\title{
Levels of three distinct p75 neurotrophin receptor forms found in human plasma are altered in type 2 diabetic patients
}

\author{
P. M. Humpert • S. Kopf • Z. Djuric • K. Laine • \\ G. Korosoglou • G. Rudofsky Jr. • A. Hamann • \\ M. Morcos • M. von Eynatten • P. P. Nawroth • \\ A. Bierhaus
}

Received: 31 January 2007 / Accepted: 16 March 2007 / Published online: 11 May 2007

(C) Springer-Verlag 2007

\begin{abstract}
Aims/hypothesis The p75 neurotrophin receptor (p75NTR) has been shown to appear in the plasma of diabetic rats, possibly indicating diabetic neuropathy. The aim of this study was to use a semi-quantitative assay for human plasma p75NTR to investigate whether this receptor is a marker of peripheral diabetic neuropathy (DPN) and autonomic cardiovascular neuropathy (CAN) in type 2 diabetic patients.

Subjects and methods Eighty type 2 diabetic patients and 25 controls without diabetes were analysed for p75NTR immunoreactivity by western blot analysis. DPN was assessed using the Neuropathy Disability Score (NDS). Cardiovascular autonomic function was detected using a standardised analysis of heart rate variability.

Results Three distinct p75NTR signals were detectable in human plasma at $\sim 75, \sim 51$ and $\sim 24 \mathrm{kDa}$, representing the full length receptor (FL) and its intracellular domain (ICD) and extracellular domain (ECD), respectively. Levels of total plasma p75NTR immunoreactivity in patients with
\end{abstract}

P. M. Humpert and S. Kopf contributed equally to this study.

P. M. Humpert $(\varangle) \cdot$ S. Kopf $\cdot$ Z. Djuric $\cdot$ K. Laine $\cdot$

G. Rudofsky Jr. A. Hamann $\cdot$ M. Morcos $\cdot$

P. P. Nawroth · A. Bierhaus

Department of Medicine I and Clinical Chemistry,

University of Heidelberg, Im Neuenheimer Feld 410,

69120 Heidelberg, Germany

e-mail: per.humpert@med.uni-heidelberg.de

G. Korosoglou

Department of Cardiology, University of Heidelberg, Heidelberg, Germany

M. von Eynatten

Department of Nephrology, Technical University Munich,

Munich, Germany type 2 diabetes were similar to those in controls. Type 2 diabetic patients had significantly higher plasma levels of ICD and lower levels of ECD. However, there were no correlations of total p75NTR immunoreactivity or ECD or ICD immunoreactivity with NDS or aspects of CAN. Conclusions/interpretation Levels of the ECD of p75NTR are reduced and levels of the ICD are increased in the plasma of type 2 diabetic patients. None of the p75NTR subunits identified in human plasma seem to be a marker of peripheral or autonomic neuronal function in patients with type 2 diabetes.

Keywords Cardiovascular autonomic neuropathy . Diabetic polyneuropathy $\mathrm{p} 75$ neurotrophin receptor . Peripheral neuropathy . Type 2 diabetes

$\begin{array}{ll}\text { Abbreviations } \\ \text { CAN } & \text { autonomic cardiovascular diabetic neuropathy } \\ \text { DPN } & \text { peripheral diabetic neuropathy } \\ \text { ECD } & \text { extracellular domain of p75NTR } \\ \text { E:I } & \text { expiration:inspiration ratio } \\ \text { FL } & \text { full-length p75NTR } \\ \text { HRV } & \text { heart rate variability } \\ \text { HsCRP } & \text { high-sensitivity C-reactive protein } \\ \text { ICD } & \text { Intracellular and transmembrane domain } \\ & \text { of p75NTR } \\ \text { P75NTR } & \text { p75 neurotrophin receptor }\end{array}$

\section{Introduction}

The p75 neurotrophin receptor has recently been shown to appear in the plasma of diabetic rats during the first 12 weeks of streptozotocin-induced diabetes, possibly 
indicating early diabetic neuropathy [1]. The p75 neurotrophin receptor (p75NTR), a member of the TNF- $\alpha$ family, was first described as a co-receptor of the Trk receptor, maintaining neuronal survival and differentiation $[2,3]$. However, its function is more sophisticated, since complexes with sortilin and Nogo receptor/LINGO1 mediate apoptosis and growth inhibition [2].

It is not known whether p75NTR appears in the plasma of humans or, if it does, whether it is an indicator of peripheral diabetic neuropathy (DPN) or autonomic cardiovascular diabetic neuropathy (CAN). Since both entities have previously been shown to be independent predictors of mortality [4, 5], the aim of the present study was to measure p75NTR immunoreactivity semi-quantitatively in patients with type 2 diabetes and to investigate correlations with different aspects of diabetic neuropathy.

\section{Subjects and methods}

Detection of p75NTR immunoreactivity in plasma samples Western blot analysis was performed as previously described [6] using a precast system (Invitrogen, Carlsbad, CA, USA). For quantification of p75NTR immunoreactivity, human albumin was depleted from plasma using an albumin depletion kit (Pall Life Sciences, Ann Arbor, MI, USA) according to the manufacturer's instructions. A $5 \mu \mathrm{l}$ aliquot of the albumin-depleted plasma sample, together with 100 and $200 \mathrm{ng}$ of recombinant p75NTR (SigmaAldrich, St Louis, MO, USA), serving as internal standard, were loaded on $10 \%$ BisTris gels (Invitrogen). Blots were blocked overnight at $4^{\circ} \mathrm{C}$ using I-Block (Tropix; Applied Biosystems, Foster City, CA, USA). The blots were incubated with p75NTR primary antibodies, directed against the extracellular domain (1:400 dilution; Biosource International, Camarillo, CA, USA) and the intracellular domain (1:7,500 dilution; Upstate, Chicago, IL, USA) for $1 \mathrm{~h}$. Secondary antibodies (Santa Cruz Biotechnologies, Heidelberg, Germany) were incubated for $45 \mathrm{~min}$ at a dilution of 1:4,000. Signals on ECL-Hyperfilm (AmershamPharmacia, Freiburg, Germany) were evaluated, calculating the mean ratio of sample to standard for both concentrations of the standard. Specificity of binding to p75NTR was confirmed by co-incubation of the membrane with primary antibody (Biosource International) and $200 \mathrm{ng}$ of the recombinant $\mathrm{p} 75 \mathrm{NTR}$ in competition experiments. For these, the ECD-binding primary antibody was incubated with excess recombinant protein for 15 min prior to incubation of the membrane as described above. A possible bias caused by loss of p75NTR immunoreactivity on the albumindepletion column was excluded by simultaneous blotting of $15 \mu l$ of plasma, albumin-depleted and eluted samples from the same subject.
Study subjects Eighty participants with type 2 diabetes were selected from patients screened for DAN and CAN in the diabetes outpatient clinic at the University of Heidelberg. Twenty-five individuals without diabetes mellitus, who served as controls, were recruited from the diabetes outpatient clinic of the University of Wurzburg (Wurzburg, Germany). All patients entered the study according to the guidelines of the local ethics committees after giving informed consent. All participants in this study had normal renal function. Type 2 diabetic patients taking tricyclic antidepressants were excluded as these medications are known to affect heart rate [7]. All routine blood parameters shown in this study were detected in the clinical laboratory of the University of Heidelberg using standardised and certified methods. GFR was calculated using the CockroftGault formula [8]. Detailed characteristics of the participants are given in Table 1.

Measurement of peripheral nervous function DPN was detected using the established neuropathy disability score as described previously [9]. The different sensory nerve functions included in this score were examined using simple bedside screening tests. Vibration perception was detected using a Rydel-Seiffer graduated tuning fork (C-64 to C-128); temperature threshold was assessed using a TipTherm device (Gesellschaft für Neurologische Diagnostik, Dusseldorf, Germany), previously described in detail [10], and pain perception was determined by the use of a pin-prick. Temperature threshold and pin-prick were tested in three different locations on the dorsum of the foot, on both feet,

Table 1 Characteristics of the study population

\begin{tabular}{lll}
\hline & $\begin{array}{l}\text { Controls } \\
(n=25)\end{array}$ & $\begin{array}{l}\text { Type 2 diabetic } \\
\text { patients }(n=80)\end{array}$ \\
\hline Age & $59 \pm 7$ & $58 \pm 6$ \\
Sex (female/male) & $4 / 21$ & $21 / 59$ \\
Diabetes duration (years) & - & $12 \pm 8$ \\
Fasting glucose (mmol/l) & $8.8 \pm 3.1$ & $5.2 \pm 0.7^{*}$ \\
HbA $_{1 \mathrm{c}}(\%)$ & $5.6 \pm 0.4$ & $7.3 \pm 1.2^{*}$ \\
Plasma creatinine ( $\mu$ mol/1) $_{\left.\text {BMI (kg/m }{ }^{2}\right)}$ & $80 \pm 18$ & $80 \pm 27$ \\
Coronary artery disease & $26 \pm 3$ & $34 \pm 6^{*}$ \\
Current smoking & 0 & 17 \\
Hyperlipidaemia & 6 & 13 \\
Systolic BP (mmHg) & 3 & 66 \\
Diastolic BP (mmHg) & $127 \pm 14$ & $137 \pm 17^{*}$ \\
Insulin therapy & $76 \pm 8$ & $82 \pm 7^{*}$ \\
Oral glucose-lowering agents & - & 49 \\
Beta-blocker & - & 59 \\
ACE/AT-II inhibitors & 3 & 39 \\
Ca ${ }^{2+}$ antagonists & 6 & 67 \\
Statins & 2 & 26 \\
& 0 & 48
\end{tabular}

Results are presented as $n$ or means \pm SD $* p<0.05$ vs controls. AT-II angiotensin II 
and were considered to be abnormal when the patient failed to detect two tests. All patients were examined by the same person to avoid intra-observer variability.

Measurements of autonomic cardiovascular function Heart rate variability (HRV) was assessed by analysis of electrocardiogram and respiration signals using a one channel electrocardiogram and a piezoelectric breathing sensor placed on the thorax. Signals were recorded at $512 \mathrm{~Hz}$, digitialised, and analysed off-line using Suempathy 100 software (Suess Medizintechnik, Aue, Germany). All participants were in a fasting state and did not smoke for at least $6 \mathrm{~h}$ prior to the recordings, which were conducted between 08.00 and $11.00 \mathrm{~h}$. HRV at rest was detected over a $5 \mathrm{~min}$ period after $5 \mathrm{~min}$ of rest in a quiet, dimmed room at constant temperature. This was followed by a $1 \mathrm{~min}$ recording of HRV while taking deep breaths ( 6 breaths/min) using an asymmetrical breathing pattern of $4 \mathrm{~s}$ inspiration and 6 s expiration; patients were given standardised breathing orders over headphones, and recordings were taken after a training phase of $\sim 30-60 \mathrm{~s}$. The ratio of expiration to inspiration (E:I) was calculated automatically by the software, using the longest $\mathrm{R}-\mathrm{R}$ interval during expiration and the shortest during inspiration. For assessment of the 30:15 ratio during a lying-to-standing challenge, the software calculated the longest $\mathrm{R}-\mathrm{R}$ interval between $\mathrm{R}$ wave 20 and 40 and the shortest between 5 and 25 while standing up. Spectral analysis electrocardiogram performed over 5 min rest was computed automatically by the software. All recordings with an excess of ectopic beats $(>3 \%)$ were excluded from the analysis. Ectopic beats were automatically corrected by extrapolation of the adaptive mean of at least two adjacent regular beats on both sides of the ectopic beat on the electrocardiogram.

Statistical analysis ANOVA and the Student's $t$ test were used for comparison of patient groups. Pearson's coefficient was calculated for correlation of p75NTR signals with patient data. Statistical evaluation was performed using SPSS 12.0 (SPSS, Chicago, IL, USA).

\section{Results}

Because of unspecific binding of the p75NTR antibodies to albumin (Fig. 1a), levels of this protein had to be removed from plasma prior to the analysis of p75NTR levels. After depletion of albumin, three specific signals could be detected at $\sim 75 \mathrm{kDa}$, representing the full length receptor (FL), at $\sim 51 \mathrm{kDa}$, representing the intracellular and transmembrane domain (ICD), and at $\sim 24 \mathrm{kDa}$, most likely representing the soluble, extracellular domain (ECD) of the receptor (Fig. 1a), which is known to be dissociated from the rest of the protein by $\alpha$-secretase activity [11]. Blotting of albumin eluted and depleted samples of subjects with different amounts of p75NTR in plasma revealed a proportional loss of $\mathrm{p} 75 \mathrm{NTR}$ on the depletion column that corresponded to the p75NTR signal obtained in the albumin-depleted material. This was indicated by the low standard deviation of the ratios of eluted to depleted samples ( $\mathrm{SD}=0.03$ for ICD and ECD signals; Fig. 1a). Specific binding of ECD was demonstrated using primary antibodies against ICD and ECD (Fig. 1b). The antibody generated against amino acids $1-160$ of ECD also recognised parts of the ICD, most likely as a result of cleavage of the receptor within or proximal to the antibody-binding site (Fig. 1b) [11]. Specificity of binding to p75NTR was further supported by co-incubation of the samples with primary antibody and excess recombinant p75NTR in a competition experiment, which led to the significant reduction of all three p75NTR signals (Fig. 1c).

The ICD signal was significantly increased in patients with type 2 diabetes compared with controls $(0.204 \pm 0.061$ vs $0.139 \pm 0.024, p<0.001)$, while ECD immunoreactivity was diminished in type 2 diabetic patients $(0.048 \pm 0.018$ vs $0.069 \pm 0.018, p<0.001$ ) (Fig. 1d). However, there were no significant differences in the FL or total p75NTR signals between patients and controls (Fig. 1d). No significant associations of ICD or FL receptor subunits with the GFR were found (data not shown), but there was a weak trend for an inverse bivariate association between the low molecular weight ECD and GFR $(R=-0.17, p=0.09)$. There was no difference in plasma ECD levels between type 2 diabetic patients receiving ACE inhibitor/angiotensin II receptor blocker therapy and those not taking this medication $(0.049 \pm 0.017$ vs $0.045 \pm 0.021, p=0.5)$. Despite previous data on the influence of diabetes and associated therapy on plasma p75NTR in rats [1], total p75NTR immunoreactivity was not correlated with fasting plasma glucose $(R=-0.08)$ or $\mathrm{HbA}_{1 \mathrm{c}}(R=0.14, p=0.21)$ in this study (Fig. 2d). The same was true for all receptor subunits (data not shown). Patients with type 2 diabetes had higher levels of plasma high-sensitivity C-reactive protein (hsCRP) than controls $(3.9 \pm 4.3$ vs $0.4 \pm 0.4 \mathrm{mg} / 1, p<$ 0.001 ), yet this marker of inflammation showed no bivariate associations with any of the p75NTR signals in the study group (not shown).

The stratification of type 2 diabetic patients into four groups affected by no, mild, moderate or severe neuronal deficits according to the Neuropathy Disability Score [9] revealed no differences between the groups in terms of plasma total p75NTR immunoreactivity (Fig. 2a). The same was true for plasma FL, ICD and ECD signals (not shown). Since p75NTR expression plays a major role in the function of sensory and sympathetic neurons in animal models [12, 13], we further studied associations with small fibre disease 

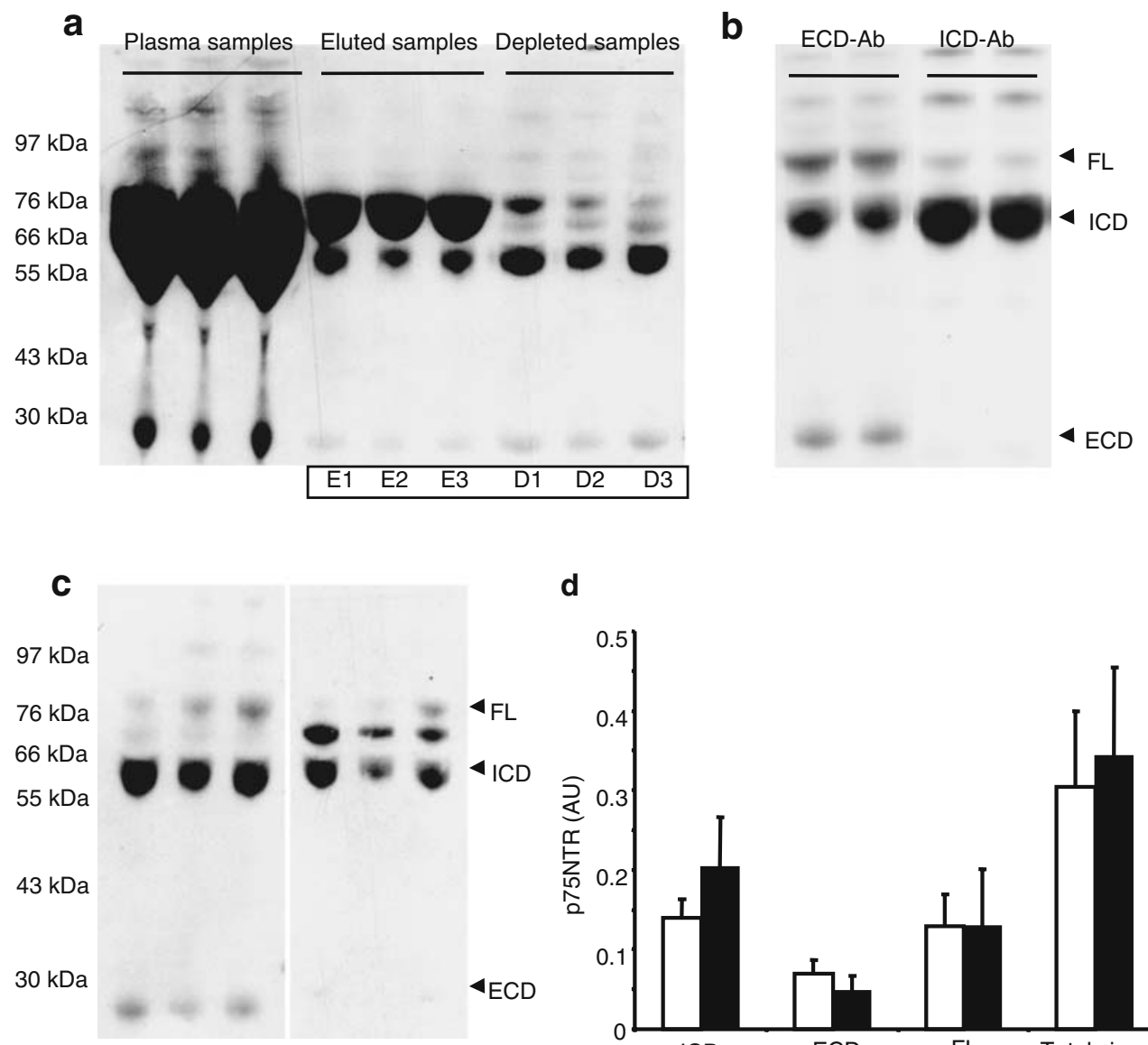

d

Fig. 1 a Due to unspecific binding of the p75NTR antibody, albumin had to be depleted from the plasma samples to allow quantification of p75NTR immunoreactivity. Incubating native plasma (left-hand side of blot) and eluted (middle of blot) and depleted (right-hand side of blot) samples it was shown in patients with different p75NTR levels that there was a loss of p75NTR on the albumin-depletion column. However, this loss was in proportion to the amount recovered, as was indicated by the low standard deviation of the ratio of eluted to depleted samples ratios in three individual participants (mean for ICD+ECD $1.34, \mathrm{SD}=0.03$ ). b Membranes were incubated with two different antibodies that bind to different receptor regions. All three p75NTR receptor signals were detected using an antibody against the ECD (ECD-Ab, left-hand side of blot), whereas the antibody directed

and different aspects of HRV as a marker of CAN. The signals for type 2 diabetic patients affected by sensory deficits (i.e. loss of temperature and/or pain detection; $n=$ 26) were similar to those for the remainder of the type 2 diabetes group for total p75NTR $(0.350 \pm 0.088$ vs $0.341 \pm 0.123)$, FL $(0.120 \pm 0.070$ vs $0.134 \pm 0.073)$, ICD $(0.201 \pm 0.053$ vs $0.206 \pm 0.066)$ and $\mathrm{ECD}(0.045 \pm 0.018$ vs $0.050 \pm 0.018)$. Bivariate correlation analysis of total p75NTR and the coefficient of HRV or the normalised lowfrequency power (a marker of sympathetic activity [14]) showed no significant associations (Fig. 2b,c). Furthermore, total p75NTR immunoreactivity was not correlated with the

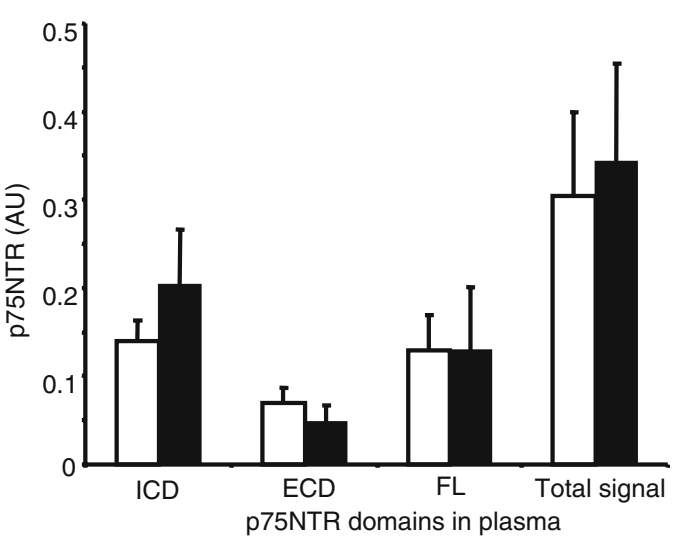

against an epitope on the ICD (ICD-Ab, right) did not detect the ECD dissociated from FL by selectase-mediated cleavage. ECD-Ab also recognised parts of the ICD signal, most likely depending on the site of extracellular cleavage. c To confirm the specificity of antibody binding, plasma samples from three separate patients were coincubated with ECD-Ab and excess recombinant p75NTR. This led to a reduction of FL, ICD and ECD signals and increased unspecific binding (right-hand side of blot) compared with samples treated with $\mathrm{ECD}-\mathrm{Ab}$ alone (left-hand side of blot). d Significant differences in p75NTR immunoreactivity between patients (black bars, $n=80$ ) and controls (white bars, $n=25$ ) were found for the ICD and the ECD (both $p<0.001$ ); FL and total p75NTR immunoreactivity did not differ significantly between the two groups

E:I ratio (a marker of parasympathetic autonomic activity; $R=0.08$ ) [14]. As shown for total p75NTR immunoreactivity, analysis of the individual signals for FL, ICD and ECD did not reveal any associations with aspects of DPN or CAN.

\section{Discussion}

This is the first study to identify the full-length receptor as well as intra- and extracellular domains of p75NTR in human plasma using specific antibodies. The subunits were previously shown in vitro to originate from cleavage of 
Fig. 2 a When type 2 diabetic patients were stratified according to the Neuropathy Disability Score into groups with no (0-2 points, $n=31$ ), mild (3-5 points, $n=18$ ), moderate (6-8 points, $n=25$ ) or severe (8-10 points, $n=6)$ peripheral neurological deficits, there were no significant differences in total p75NTR immunoreactivity between the groups by ANOVA. In addition, bivariate correlations of total p75NTR with the coefficient of HRV $(R=0.06)$

(b), the normalised lowfrequency power of HRV (reflecting sympathetic activity) $(R=0.06)(\mathbf{c})$ and $\mathrm{HbA}_{1 \mathrm{c}}$ as a measure of long-term glucose control $(R=0.14)(\mathbf{d})$ showed no significant associations a

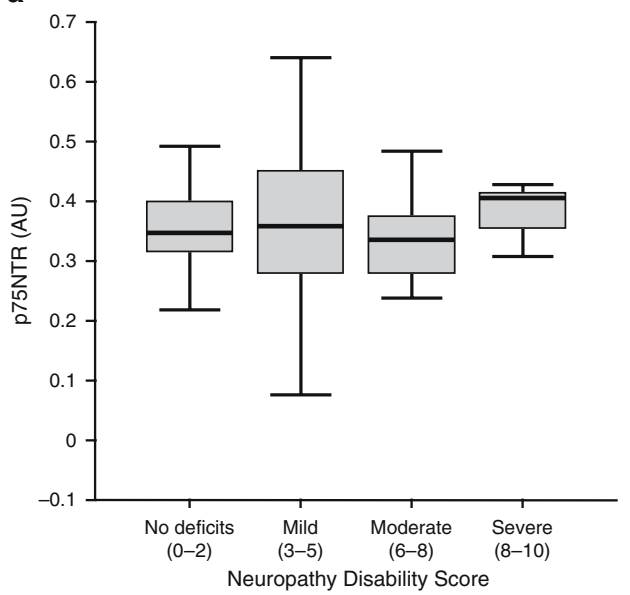

C

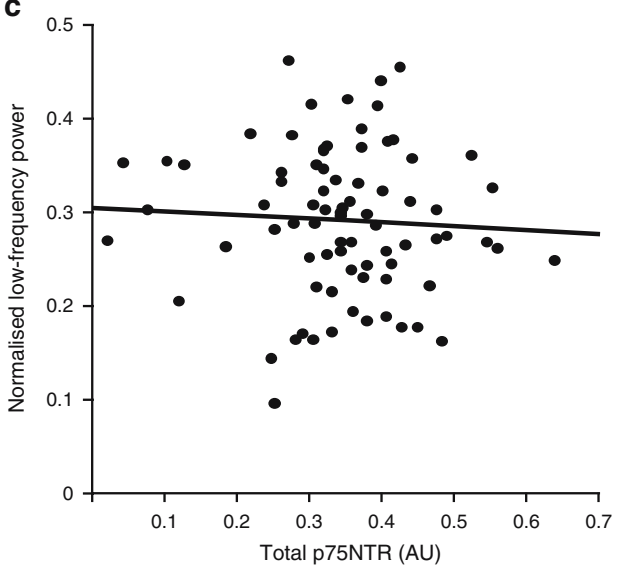

b

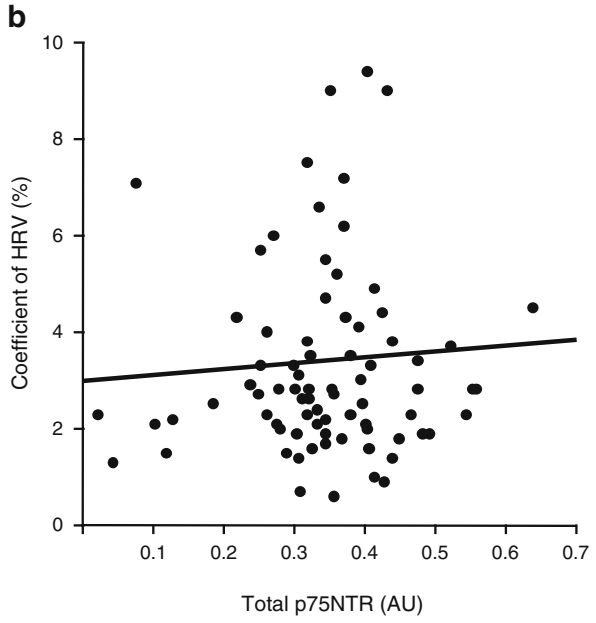

d

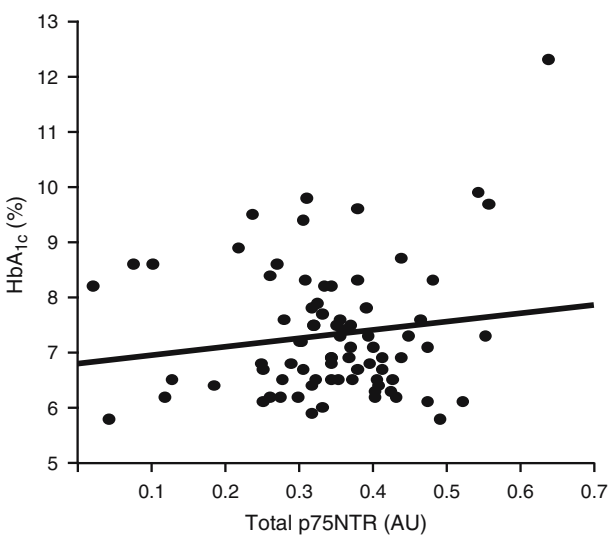

the p75NTR ectodomain [11]. The role of $\mathrm{p} 75 \mathrm{NTR}$ as a proapoptotic receptor implies that decreased expression of this protein leads to enhanced survival of damaged neurons, as shown in p75NTR knockout mice [15]. However, the immunoreactivity of both the full-length receptor and total p75NTR was similar between patients and controls; a finding that does not support an increased rate of neuronal apoptosis. ICD immunoreactivity was increased and ECD immunoreactivity was decreased in the plasma of type 2 diabetic subjects compared with controls. In vitro studies have shown that $\alpha$-and $\gamma$-secretase activity leads to cleavage of p75NTR, followed by a release of the ICD into the cytoplasm [16]. This triggers an intracellular signalling cascade, activating the proinflammatory and antiapoptotic transcription factor nuclear factor-kB [16], which plays a major role in the activation of an inflammatory state in different cell lines in hyperglycaemia and experimental diabetic neuropathy $[6,17,18]$. Although the type 2 diabetic patients who participated in this study had a proinflammatory phenotype, as indicated by increased levels of hsCRP, none of the p75NTR domains were found to be correlated with hsCRP. Since p75NTR exhibits pro-and antiapoptotic, as well as proinflammatory properties depending on cell type, co-receptor expression and growth factor binding (reviewed in [19]), no definite conclusions can be drawn from the significant alterations in levels of the plasma p75NTR subunits in diabetic patients compared with controls. Future experimental and clinical studies on the influences of hyperglycaemia, inflammation and oxidative stress on p75NTR expression and cleavage, as well as receptor-dependent cellular changes, will be needed to establish the importance of our finding.

Although several screening tests have been defined as reliable for the diagnosis of DPN and CAN, there is no blood test available that specifically indicates the individual risk of disease progression or the development of associated complications. Since the diagnosis of CAN relies on the detection of variability in the $\mathrm{R}-\mathrm{R}$ interval under haemodynamic stress (i.e. deep breathing, Valsalva manoeuvre, lying to standing) [20], a variety of external factors, such as medication, circadian rhythm and age, can cause bias [20]. In a nerve crush model in diabetic mice, p75NTR appears in plasma [1] and plays a role in the switch from adrenergic stimulation of cardiomyocytes to inhibitory cholinergic transmission [21]. 
Hence, plasma p75NTR might be a blood marker for DPN and CAN that allows early diagnosis and prediction of associated complications. The results of this study do not provide evidence in support of this hypothesis, as plasma p75NTR immunoreactivity did not serve as a marker of DPN or CAN in the type 2 diabetic patients. Our data are based on a semi-quantitative measurement, and development of advanced analytical methods using specific antibodies might allow more accurate measurement of plasma p75NTR concentrations. However, it seems doubtful that differences in plasma p75NTR between patients affected by no or severe peripheral neuronal deficits in the Neuropathy Disability Score not detected by the immunoassay presented here (Fig. 2a), will be of interest for clinical assessments.

Remarkably, p75NTR was not associated with long-term glucose control or fasting plasma glucose. It is therefore unlikely that the p75NTR expression is modulated by glucose levels. However, the data presented do not exclude the possibility that plasma levels of p75NTR are modulated by glucose levels at an early stage of disease or in type 1 diabetes, as was shown in the streptozotocin-induced diabetes rat model [1]. Since this was not the focus of our experimental design, a clinical study on type 1 diabetes or on patients after pancreatectomy would need to be conducted to address this question.

In conclusion, this is the first report to show the appearance of full-length p75NTR together with the separate intra- and extracellular domains of the receptor in human plasma. No associations of p75NTR receptor subunits with aspects of DPN or CAN were found in the type 2 diabetic patients studied. Further clinical and experimental studies should be conducted to elucidate the biological significance of altered ICD and ECD plasma immunoreactivity in type 2 diabetes.

Acknowledgements Part of this work was supported by the Manfred Lautenschläger Foundation (to P. P. Nawroth) and the Juvenile Diabetes Research Foundation (to A. Bierhaus and P. P. Nawroth).

Duality of interest statement The authors declare that there is no duality of interest associated with this study.

\section{References}

1. Chilton L, Middlemas A, Gardiner N, Tomlinson DR (2004) The p75 neurotrophin receptor appears in plasma in diabetic ratscharacterisation of a potential early test for neuropathy. Diabetologia 47:1924-1930

2. Barker PA (2004) p75NTR is positively promiscuous: novel partners and new insights. Neuron 42:529-533

3. Barker PA, Shooter EM (1994) Disruption of NGF binding to the low affinity neurotrophin receptor p75LNTR reduces NGF binding to TrkA on PC12 cells. Neuron 13:203-215
4. Cusick M, Meleth AD, Agron E et al (2005) Associations of mortality and diabetes complications in patients with type 1 and type 2 diabetes: early treatment diabetic retinopathy study report no. 27. Diabetes Care 28:617-625

5. Maser RE, Mitchell BD, Vinik AI, Freeman R (2003) The association between cardiovascular autonomic neuropathy and mortality in individuals with diabetes: a meta-analysis. Diabetes Care 26:1895-1901

6. Bierhaus A, Schiekofer S, Schwaninger M et al (2001) Diabetesassociated sustained activation of the transcription factor nuclear factor-kappaB. Diabetes 50:2792-2808

7. Roose SP, Laghrissi-Thode F, Kennedy JS et al (1998) Comparison of paroxetine and nortriptyline in depressed patients with ischemic heart disease. JAMA 279:287-291

8. Sampson MJ, Drury PL (1992) Accurate estimation of glomerular filtration rate in diabetic nephropathy from age, body weight, and serum creatinine. Diabetes Care 15:609-612

9. Young MJ, Boulton AJ, MacLeod AF, Williams DR, Sonksen PH (1993) A multicentre study of the prevalence of diabetic peripheral neuropathy in the United Kingdom hospital clinic population. Diabetologia 36:150-154

10. Ziegler D, Siekierka-Kleiser E, Meyer B, Schweers M (2005) Validation of a novel screening device (NeuroQuick) for quantitative assessment of small nerve fiber dysfunction as an early feature of diabetic polyneuropathy. Diabetes Care 28:1169-1174

11. Zampieri N, Xu CF, Neubert TA, Chao MV (2005) Cleavage of p75 neurotrophin receptor by alpha-secretase and gammasecretase requires specific receptor domains. J Biol Chem 280:14563-14571

12. Lee KF, Bachman K, Landis S, Jaenisch R (1994) Dependence on p75 for innervation of some sympathetic targets. Science 263:1447-1449

13. Lee KF, Li E, Huber LJ et al (1992) Targeted mutation of the gene encoding the low affinity NGF receptor p75 leads to deficits in the peripheral sensory nervous system. Cell 69:737-749

14. Therapeutics and Technology Assessment Subcommittee of the American Academy of Neurology (1996) Assessment: clinical autonomic testing report of the Therapeutics and Technology Assessment Subcommittee of the American Academy of Neurology. Neurology 46:873-880

15. Sorensen B, Tandrup T, Koltzenburg M, Jakobsen J (2003) No further loss of dorsal root ganglion cells after axotomy in p75 neurotrophin receptor knockout mice. J Comp Neurol 459:242-250

16. Kanning KC, Hudson M, Amieux PS, Wiley JC, Bothwell M, Schecterson LC (2003) Proteolytic processing of the p75 neurotrophin receptor and two homologs generates C-terminal fragments with signaling capability. J Neurosci 23:5425-5436

17. Schiekofer S, Andrassy M, Chen J et al (2003) Acute hyperglycemia causes intracellular formation of CML and activation of ras, p42/44 MAPK, and nuclear factor kappaB in PBMCs. Diabetes 52:621-633

18. Bierhaus A, Haslbeck KM, Humpert PM et al (2004) Loss of pain perception in diabetes is dependent on a receptor of the immunoglobulin superfamily. J Clin Invest 114:1741-1751

19. Schor NF (2005) The p75 neurotrophin receptor in human development and disease. Prog Neurobiol 77:201-214

20. Vinik AI, Maser RE, Mitchell BD, Freeman R (2003) Diabetic autonomic neuropathy. Diabetes Care 26:1553-1579

21. Yang B, Slonimsky JD, Birren SJ (2002) A rapid switch in sympathetic neurotransmitter release properties mediated by the p75 receptor. Nat Neurosci 5:539-545 